\title{
A Continuous Developmental Model for Wind Farm Layout Optimization
}

\author{
Dennis Wilson \\ CSAIL - MIT \\ 32 Vassar Street \\ Cambridge, MA 02139, USA \\ dennisw@mit.edu
}

\author{
Sylvain Cussat-Blanc \\ University of Toulouse \\ IRIT - CNRS - UMR5505 \\ 21 allée de Brienne \\ 31042 Toulouse, France \\ cussat@irit.fr
}

\author{
Kalyan Veeramachaneni \\ CSAIL - MIT \\ 32 Vassar Street \\ Cambridge, MA 02139, USA \\ kalyan@csail.mit.edu
}

\author{
Una-May O'Reilly \\ CSAIL - MIT \\ 32 Vassar Street \\ Cambridge, MA 02139, USA \\ unamay@csail.mit.edu
}

\author{
Hervé Luga \\ University of Toulouse \\ IRIT - CNRS - UMR5505 \\ 21 allée de Brienne \\ 31042 Toulouse, France \\ luga@irit.fr
}

\begin{abstract}
We present Devo-II, an improved cell-based developmental model for wind farm layout optimization. To address the shortcomings of discretization, DEvo-II's gene regulatory networks control cells that act in a continuous rather than discretized grid space. We find that DEvo-II is competitive, and in some cases, superior with respect to stateof-the-art global, stochastic search approaches when a suite of algorithms is evaluated on different wind scenarios. The modularity of the genetic regulatory network computational paradigm in terms of isolating its search algorithm, the regulatory network simulation and the cell simulation, allowed this improvement to largely focus upon cell simulation. This indicates a robustness property of the paradigm's design. As well, wind farm layout optimization highlights how developmental models can be considered more efficient than other optimization methods because of their "optimize once, usemany" adaptability.
\end{abstract}

\section{Keywords}

Layout optimization; Developmental model; Gene regulatory network; Machine learning

\section{INTRODUCTION}

Wind farm layout optimization is a complex problem and recent growth of large wind farms has increased demands on designers. Typically, the problem has been cast as a geometric optimization problem, usually on a discrete grid, using a power-based cost function. Direct search approaches have been employed wherein specific constraints of the problem are translated into a predefined fitness function and conditions, e.g. wind speed data and farm dimensions, are inputs. Once this configuration of the algorithm is set up by the wind farm designer, the optimization takes hours or days to converge, and must be run again if the problem changes.

We present a novel approach, DEvo-II, that facilitates wind farm optimization where, once a developmental model is evolved, results are available in a matter of seconds regardless of different constraints and conditions. In [23], we designed a discrete cell-based developmental model, DEVO-I, and obtained encouraging results. This paper, with DEvoII, represents an improvement on DEVO-I, by casting the developmental model in a continuous space. In general, a cell-based developmental model grows a wind farm layout using cells as an analog for turbines. The cells are each controlled by a gene regulatory network which is trained using a genetic algorithm to find the best turbine efficiency and the best number of turbines. The cells respect the local and global constraints and attempt to position themselves optimally in a representation of the farm environment. To do so, each cell senses the wind coming from various directions and decides what to do: divide, reorient its division or migration heading, migrate, grow, or die. In DEvo-I our farm representation was discrete. With DEvo-II we investigate whether the continuous developmental model produces layouts with comparable quality to state-of-the-art approaches.

\section{WIND FARM LAYOUT OPTIMIZATION PROBLEM}

The wind farm layout optimization problem is to identify turbine positions in 2-D plane, $x, y$ coordinates, such that the energy capture is maximized while costs associated with a number of other factors are minimized. The energy capture for a turbine takes into account the following:

Wind Scenario: Wind speed, $v$, represented as a random variable with a Weibull distribution that is a sum- 


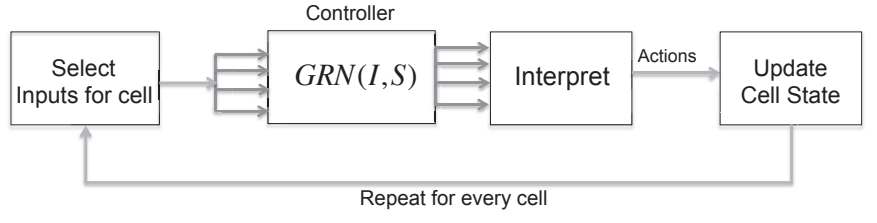

(a) A developmental step

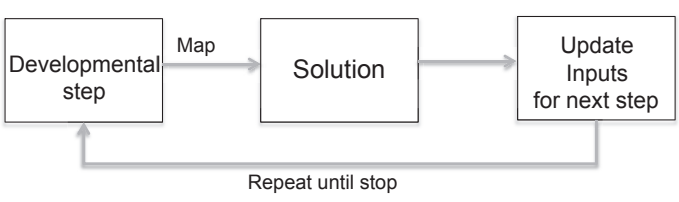

(b) Generation of a solution

Figure 1: Two components of the developmental approach

mary of wind speed at that location for a period of time. This is given by $p_{v}(v ; c, k \mid \theta)$, where $c$ and $k$ are Weibull shape and scale parameters and $\theta$ is a wind directional bin. The wind speed distribution is different for different directional bins, $\theta$. Additionally, wind flows from a certain direction with some probability $p(\theta)$. Together $p_{v}(v ; c, k \mid \theta)$ and $p(\theta)$ are referred to as wind resource/scenario in this paper.

Power curve: A function $\eta(v)$, known as a power curve, gives the power generated by a turbine for the given wind speed $v$. The power curve is dependent on the turbine make and model.

Wake effects: In a particular directional bin, for a given turbine $i$ located at $x_{i}, y_{i}$ a number of other turbines affect the wind it experiences. This is called wake effect. Given other turbines locations $x_{j}, y_{j}$ for all $j \in\{1 \ldots i-1, i+1 \ldots n\}$, the turbines that affect the particular turbine is determined using a wake model. This is documented in [14]. If a turbine located at $x_{i}, y_{i}$ is in the wake of another turbine located at $x_{j}, y_{j}$ in a given direction, the wind speed distribution experienced by the turbine is modified by changing the parameter $c$ resulting in a turbine specific $c_{i} \mid \theta$. The value $c_{i}<c$ is reduced in proportion to the euclidean distance between $i$ and $j$.

To evaluate the energy capture the objective function calculates the expected value of the energy capture for a given wind resource and turbine positions. For a single turbine at position $\left(x_{i}, y_{i}\right)$, it first determines its modified wind resource for each directional bin based on other turbine positions and then calculates its energy capture using:

$$
E=\int_{\theta} p(\theta) \int_{v} p_{v}^{\theta}\left(v, c_{i}, k_{i} \mid \theta\right) \eta(v)
$$

Equation 1 evaluates the overall average energy over all wind speeds for a given wind direction, and then averages this energy over all wind directions. Energy is calculated for every turbine and then summed together to give global energy capture. To implement a wake model and reproduce the results there are three options as described in [14] and [19]. ${ }^{1}$

To evaluate the efficiency of a wind field, the energy capture is compared to the theoretical maximum energy possible by as many turbines, if they were free of energy decreasing wake effects, resulting in a wake free ratio:

\footnotetext{
${ }^{1}$ Software that evaluates energy capture given turbine positions and wind resource is available from https:// github.com/d9w/WindFLO. A competition being organized at GECCO 2014 will result in an open source software release for standardized comparisons.
}

$$
R_{w f}=\frac{E_{t o t}}{E_{w f} * n}
$$

where $R_{w f}$ is the wake free ratio, $E_{t o t}$ is the layout energy output, $E_{w f}$ is the theoretical maximum energy output of one turbine and $n$ is the number of turbines in the layout.

In situations where evaluation of both the efficiency, given by the wake free ratio, and the number of turbines in a field is necessary, in this paper we use the following fitness function:

$$
f= \begin{cases}R_{w f} & \text { if } 400 \leq n \leq 600 \\ R_{w f} * \frac{n}{400} & \text { if } n<400 \\ R_{w f} * \frac{1200-n}{600} & \text { if } n>600\end{cases}
$$

where $R_{w f}$ is the wake free ratio of the layout and $n$ is the number of the turbines in the layout. This could be easily replaced by an economic model that pits energy capture against turbine cost.

\section{DEVELOPMENTAL APPROACH}

In this paper, we develop an approach that works differently than a direct global search method. Our solution to the optimization problem $\operatorname{argmax}_{X} f(X)$ consists of three components:

1. Definition of a developmental step: As shown in Figure 1 (a), the model is defined for entities termed cells as characterized by their state $S$. A multi-input, multi-output cell controller (GRN in this paper) processes a cell's current state information and inputs $I$ derived for the cell to generate its outputs. The interpreter uses these outputs to create actions for the cell and updates the state of the cell. This process is repeated for every cell and is known as a developmental step.

2. Generation of a solution At the end of each developmental step a solution is generated and the inputs for the controller (on a per cell basis) are derived from the solution. The developmental steps are repeated till a stopping criterion is met. The solution at the conclusion of the last developmental step is the final solution for the design/optimization problem.

3. Learning the controller structure and parameters: The problem of optimization then becomes that of learning (or optimizing) the structure and parameters for the controller such that when the developmental process is run it produces the best solution for the problem. When the optimization problem is specified by its scenarios, the developmental model is run for multiple scenarios and average performance is considered when evaluating a controller's efficacy. 
Once a controller is learnt, when a new instance of the optimization problem is encountered, the developmental process as described in components 1 and 2 are executed to generate a solution. Note the optimization component (3) does NOT have to be repeated. This is the computational advantage of the developmental approach over any global optimization procedure. Another advantage is that the dimensionality of the controller design problem only scales with its inputs and outputs and not with the dimensionality of the original optimization problem. The efficacy of this methodology is however dependent on the definition of the cell, state, and its actions, the inputs for the controller and its outputs and how they are interpreted. In this paper, we focus on developing these for the wind farm layout optimization problem.

\section{LAYOUT OPTIMIZATION - DEVELOP- MENTAL MODEL}

As explained in the previous section, for the developmental approach we define the cell structure, the state of the cell, the inputs for the controller, the space of outputs and actions of the cells and the mapping between controller outputs and actions of the cell. In this section we describe each of these for the layout optimization problem. In next section we describe the controller followed by a methodology to learn the controller parameters and structure.

Cell definition and State: The cell is a circular structure and a turbine is placed at its center. The state of a cell is defined by 5 attributes $-\theta_{m}, \theta_{d}, r$, cen and $b . \theta_{m}$ and $\theta_{d}$ are continuous values between 0 and $2 \pi$ that represent the migration and division directions, respectively. $r$ is a continuous value between $R / 2$ and $R$, where $R$ is the minimum possible distance between any two wind turbines, called the security distance, and $r$ represents the cell's radius. Therefore, two cells with minimum radii of $R / 2$ each would be $R$ apart and would not be within the security distance. cen is a vector of two continuous values, $x_{c}$ and $y_{c}$, that represent the position of the center of the cell, and therefore the turbine, and are constrained to $[0, w]$ and $[0, h]$, respectively, where $w$ and $h$ are the width and height of the farm. The last state variable, $b$, is simply a boolean value indicating whether or not the cell is "alive". Dead cells, those with $b=$ False, are removed from the layout at the end of each developmental step.

Inputs: The key elements that control the behavior of the cell (which we define below) are the inputs and outputs for the gene regulatory network (the controller). DEvo-II's gene regulatory network uses five inputs. For each cell, the

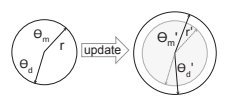

(a) Update

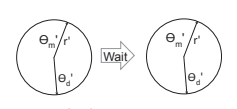

(b) Wait

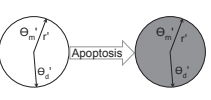

(c) Apoptosis

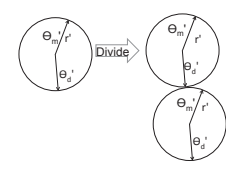

(d) Divide

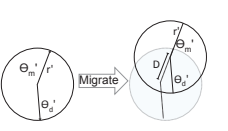

(e) Migrate
Figure 2: Different actions for the cells. wind energy that could be harnessed is calculated for each directional bin. Note that this is influenced by the other turbines in the field (also, positions of other cells). For each cell, the total energy captured at its center is also calculated (the sum of energy capture in all directional bins). Let $\max _{e}$ be the maximum energy capture for any single directional bin among all cells, and let $\max _{E}$ be the maximum total energy capture among all cells. The inputs for a cell are calculated as follow:

1. the maximum energy capture at cen divided by the maximum energy capture for a single directional bin found by any cell, $\max _{e}$,

2 . the direction with the maximum energy capture, divided by $2 \pi$,

3 . the opposite direction of the maximum energy capture by $2 \pi$,

4. the total energy capture for the cell divided by the maximum total energy capture found by any cell, $\max _{E}$.

5. the percentage of area covered by other cells within a circle of radius $4 R$ from cen.

These input proteins are non-trivially different from their predecessors in Devo-I because they are relative, not absolute. To compute these inputs, we use a wind model, described in $\S 2$, that takes into account the inter-turbine interferences. From the initial wind distribution provided by the wind scenario, this model computes the wake generated by the turbines and therefore their energy capture, which allows the relative quantities in 1-4 to be calculated.

Outputs and actions: For our problem we define 12 outputs for the GRN. Four actions are defined for the cell as shown in Figure 2. The interpreter uses the outputs and performs the following steps.

\section{Update State:}

The cell state (orientation vectors and radius) are updated using the concentrations of the proteins $o_{1}, o_{2}$, $O_{3}, O_{4}, O_{5}, O_{6} . O_{1}$ and $O_{2}$ are used to update the orientation for division, $\theta_{d}$, by $\theta_{d}=\theta_{d}+\left(o_{1}-o_{2}\right) /\left(o_{1}+o_{2}\right)$. $o_{3}$ and $o_{4}$ are used to update the orientation for migration, $\theta_{m}$, by $\theta_{m}=\theta_{m}+\left(o_{3}-o_{4}\right) /\left(o_{3}+o_{4}\right)$. To optimize their energy capture, the cells can also modify their radii in order to compact or to expand their structure. A cell can increase or decrease its radius, $\mathrm{r}$, by $r=r+\left(o_{5}-o_{6}\right) /\left(o_{5}+o_{6}\right)$, at each developmental step, within the radius limit $[170.5,310]$. The minimum value corresponds to the turbine security distance. The maximum one has been chosen empirically to keep the distance between the turbines consistent. The process of updating the state of the cell is depicted in Figure 2(a).

2. Choose an action: 4 outputs $o_{7} \ldots o_{10}$ function as cell action selectors. Each represents a different action and the action of the one with the highest concentration is selected. We list below the four actions.

(a) Divide: if divide is chosen the cell creates a new cell adjacent to it $2 r$ apart from its own center in the direction of the orientation $\theta_{d}$ (as shown in Figure $2(\mathrm{~d})$ ). The state of the new cell is the same as the current cell. When the layout is formed it is assumed that a turbine is at the center of new cell.

(b) Wait: if wait is chosen the cell does not do anything (as shown in Figure 2(b)). 
(c) Apoptosis: if apoptosis is chosen the value $b$ in the state is changed to 0 implying that this cell no longer exists.

(d) Migrate: if migrate is chosen, the cell's center is moved in the direction of $\theta_{m} .2$ output proteins $o_{11}$ and $o_{12}$ are used to determine the distance by which the cell is moved. It is given by $v_{m}$ : $v_{m}=r * o_{11} /\left(o_{11}+o_{12}\right)$ where $r$ is the cell radius (as shown in Figure 2(e)).

\section{Developmental process and stopping criteria}

In each developmental step of the layout growth, each cell updates its radius (size), migration and division directions, chooses an action, and then executes that action. To ensure the turbine security constraint is not violated, a massspring-damper system is used [13]. When two cells overlap, a spring links their centers and the mass-spring-damper system repulses the cells along the line joining them while the dampers reduce the possible oscillatory behaviors generated by the springs.

The mass-spring-damper system is run until all constraints are resolved. Mass-spring-damper systems are often uses in artificial developmental model $[8,5,17]$ because they are simple and efficient models to simulate cellular dynamics. For our problem, they help constrain the developmental process. After dampening all the cells that, either by division, migration or due to the mass-spring-damper dynamics, move into an invalid position (outside the layout or on an obstacle) are deleted from the layout. This ensures the validity of the final layout.

One last key element of the developmental model is how development is deemed to be complete. One of our stop criterions measures stability to determine the end of the developmental process. After five developmental steps which initiate cell proliferation, the developmental process continues while at least one cell is dividing or moving, either by migration or due to resizing. Another stop criterion caps development at a maximum number of steps (proportional to farm size).

\section{A CELL'S CONTROLLER: GRN}

As defined in the previous section, the outputs of the GRN control the functioning of an individual cell. In this section we describe the design of the $G R N(I, S)$. In nature, a gene regulatory network (GRN) is a network of proteins that controls the behavior of the cells. In a living organism, a cell has several functions described in its genome. A gene regulatory network controls their expressions by the use of external signals collected from protein sensors localized on the membrane [4]. These signals activate or inhibit the transcription of the genes, which then determines the cell's behavior.

In our model, a similar network of proteins is optimized in order to generate the simulated cells' behaviors. This kind of controller has been used in many developmental models of the literature $[10,5,2]$ and to control virtual and real robots $[16,11,3]$.

The GRN model used in this work is a simplified computational model of a real gene regulatory network. It has been designed for computational purposes and not to simulate protein interactions. In it, a gene regulatory network is defined as a set of interacting proteins. Each protein has the following properties:
- The protein identifier, encoded as an integer between 0 and $\varepsilon . \varepsilon$ (here equal to 32) can be changed in order to control the precision of the GRN.

- The enhancer identifier, encoded as an integer between 0 and $\varepsilon$. The enhancer identifier is used to calculate the enhancing matching factor between two proteins.

- The inhibitor identifier, encoded as an integer between 0 and $\varepsilon$. The inhibitor identifier is used to calculate the inhibiting matching factor between two proteins.

- The type, which determines if the protein is an input protein, whose concentration is given by the environment of the GRN and whose regulates other proteins but is not regulated; an output protein, whose concentration is used as an output of the network and which is regulated but does not regulate other proteins; or a regulatory protein, an internal protein that regulates and is regulated by other proteins.

The dynamics of the GRN are calculated as follows. First, the affinity of a protein $a$ with another protein $b$ is given by the enhancing factor $u_{a b}^{+}$and the inhibiting $u_{a b}^{-}$:

$$
u_{a b}^{+}=\varepsilon-\left|e n h_{a}-i d_{b}\right| ; u_{a b}^{-}=\varepsilon-\left|i n h_{a}-i d_{b}\right|
$$

where $i d_{j}$ is the identifier, $e n h_{j}$ is the enhancer identifier and $i n h_{j}$ is the inhibitor identifier of protein $j$.

Then, the proteins are compared two by two using the enhancing and the inhibiting matching factors. For each protein of the network, the global enhancing and inhibiting values are given by the following equations:

$$
g_{i}=\frac{1}{N} \sum_{j}^{N} \phi_{j} e^{\beta\left(u_{i j}^{+}-u_{\max }^{+}\right)} \quad ; \quad h_{i}=\frac{1}{N} \sum_{j}^{N} \phi_{j} e^{\beta\left(u_{i j}^{-}-u_{\max }^{-}\right)}
$$

where $g_{i}$ (resp. $h_{i}$ ) is the enhancing (resp. inhibiting) value for a protein $i, N$ is the number of proteins in the network, $\phi_{j}$ is the concentration of protein $j$ and $u_{\max }^{+}$(resp. $u_{\max }^{-}$) is the maximum enhancing (resp. inhibiting) matching factor observed. $\beta$ is a control parameter described hereafter.

The final modification of protein $i$ concentration is given by the following differential equation:

$$
\frac{d \phi_{i}}{d t}=\frac{\delta\left(g_{i}-h_{i}\right)}{N_{\phi}}
$$

where $N_{\phi}$ is a function that normalizes the output and regulatory protein concentrations to sum to 1 .

$\beta$ and $\delta$ are two constants that set up the speed of reaction of the regulatory network. The higher these values, the more sudden the transitions in the GRN. The lower they are, the smoother the transitions.

\section{LEARNING THE GRN}

The GRN is encoded in a genome to be evolved by a standard genetic algorithm. The genome contains two independent chromosomes. The first is a variable length chromosome of indivisible proteins. Each protein is encoded within three integers between 0 and $\varepsilon$ for the three different identifiers: input, output, and regulatory. The variation operators of a standard GA are redefined. Crossover consists of exchanging subparts of two different networks. Because proteins are indivisible, the crossover points have to be chosen between two proteins. This ensures the integrity of each 


\begin{tabular}{|c|c|}
\hline Population size & 500 \\
\hline Mutation rate & $10 \%$ \\
\hline Crossover rate & $75 \%$ \\
\hline \multirow{2}{*}{ Selection } & 3-player tournament \\
& with elitism \\
\hline \multirow{3}{*}{ Minimum GRN size } & 5 input proteins + \\
& 12 output proteins + \\
& 15 regulatory proteins \\
\hline \multirow{3}{*}{ Maximum GRN size } & 5 input proteins + \\
& 12 output proteins + \\
& 50 regulatory proteins \\
\hline
\end{tabular}

Table 1: Parameters of the genetic algorithm used to train the GRN.

sub-network, and the local connectivity is maintained; only new links between the different sub-networks are created. Mutation is applied in three equally probable ways: $m u$ tating an existing protein by randomly changing one of its three integers, adding a new protein randomly generated or removing one protein randomly chosen in the network.

The coefficients $\beta$ and $\delta$ presented in the dynamics model are encoded in a second independent chromosome which contains only these values. They are coded with doubleprecision floats in $[0.5 ; 2]$ (empirically chosen).

In this work, we have used a master-worker model distributed genetic algorithm in order to reduce the optimization duration. Table 1 presents the parameters used to evolve the gene regulatory network in this experience.

As the developmental process is completely deterministic, running the developmental process only once is sufficient to calculate the fitness of the corresponding wind farm layout. However, to avoid over-specialization on one scenario, this procedure is repeated on three different scenarios and the minimum fitness of all three scenarios is kept as the final fitness. The wind scenarios used to train the regulatory network are described in the supplementary materials as scenarios 1-3, and are available for download as well as part of the aforementioned open source software.

\section{COMPARATIVE STUDY}

We compare Devo-II to a set of state-of-the-art layout optimization techniques: a genetic algorithm (GA), Particle

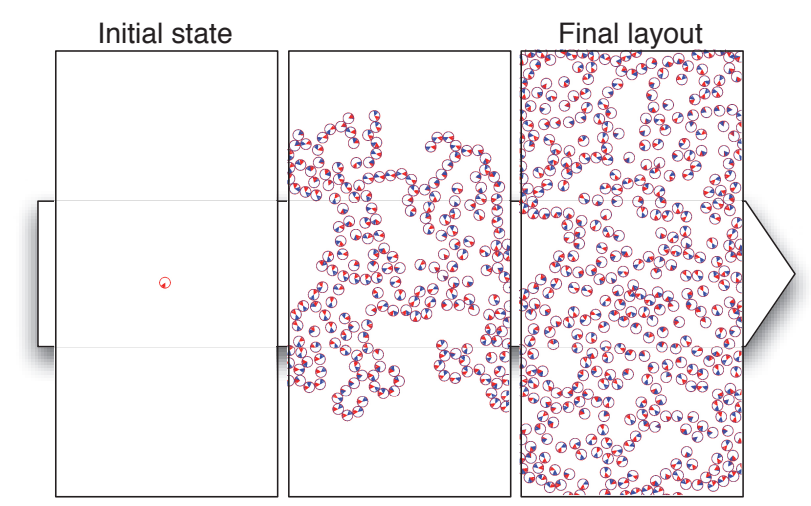

Figure 3: Example of development of a layout, showing the first, middle, and last developmental steps.
Swarm Optimization (PSO), and the Turbine Distribution Algorithm (TDA). We also compare Devo-II to Devo-I. After briefly explaining these techniques, which are further detailed in $[12,20,23]$, this section presents these comparisons.

\subsection{Existing approaches}

\subsubsection{Genetic algorithm (GA)}

Genetic algorithms are commonly used to optimize wind farm layout. The farm area is discretized with a grid $[15,7$, $9,21,6,18,24]$. The size of each cell of the grid corresponds to the minimal security distance between 2 turbines. The genetic algorithm then optimizes a binary genome in which each gene represents the presence or absence of a turbine in each cell of the grid. This approach has the advantage of optimizing the number of turbines, but the position of the turbines is not precisely optimized as they are limited to the center of the cell. In the scope of this study, we have implemented our own genetic algorithm. The fitness function used to evaluate each layout is the one presented in equation 2. The genetic algorithm is set up with a population size of 500 individuals, a 3-player tournament selection with elitism, $20 \%$ of mutation and $70 \%$ of crossover. The genetic algorithm is run for 500 generations, which is sufficient to converge.

\subsubsection{Particle swarm optimization (PSO)}

In the particle swarm optimization approach, a particle represents a turbine layout by a vector of $\mathrm{N}(\mathrm{x}, \mathrm{y})$ Cartesian coordinates, where $\mathrm{N}$ is the maximum number of turbines[22, 1, 19]. In other words, the PSO search space has $2 \mathrm{~N}$ dimensions, $\mathrm{N}$ being the maximum possible number of turbines. The particle also maintains a memory of the global best position already found in a population of particles. For each iteration of the algorithm, the particles' velocities are calculated according to their own fitness, the position of the current best solution, and nearby particles' positions. The next positions of the particles can be immediately deduced from the velocities. A constraint-repairing algorithm, described in [19], is used to meet the security distance constraint. In this method, starting with an initial turbine, a turbine is added to a layout at position proposed by the particle only if it is not within the security distance to any existing turbine. The PSO is run with each particle on a different thread with a maximum of 1000 turbines, 20 particles, a neighborhood size of 3 , and the fitness function 2 . The constraint reparation mechanism causes many of the turbines to not enter the layout; 1000 as a maximum was determined empirically to place 400 turbines. The PSO is run for 100 iterations or equivalently 2000 layout evaluations, which is sufficient to converge.

\subsubsection{Turbine Distribution Algorithm (TDA)}

TDA uses randomized modification of turbine location starting with an initial layout [20]. At each iteration, the best layout is modified by slightly modifying a randomly chosen turbine in the layout and perturbing it to move it away from its closest neighbors in order to minimize the local interferences produced by the turbines. The new layout is compared to the previous best layout using energy output. This process is repeated a sufficient number of iterations to reach an optimal layout. This approach has the advantage of 


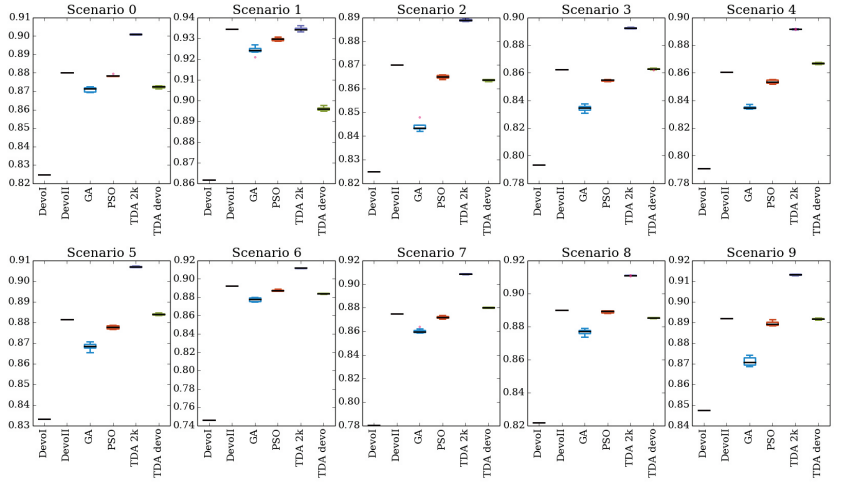

Figure 4: Results of the comparative study of layout optimization approaches. Each approach has been evaluated 8 times on each scenarios.

precisely positioning the turbines in the layout because each turbine position is computed with a high resolution but it requires a high number of layout evaluation (which is computationally expensive) and only works with a fixed number of turbines. This approach is, to our knowledge, the current best approach to optimize a layout with a fixed number of turbines. We have used the Wagner \& al. implementation of TDA $[20]^{2}$, with the number of turbines determined by Devo-II. First, we run TDA for 2000 iterations, as done in [20], to determine an optimal layout. Then, we compare TDA running for the same number of evaluations as DEvoII to provide a balanced comparison based on runtime.

\subsubsection{Discrete developmental model (Devo-I)}

Devo-I is very similar to Devo-II: cells are controlled by a gene regulatory network to populate a 2-D layout. They use wind velocity input to decide their action: divide, rotate their division clockwise or counterclockwise, wait and die. However, instead of populating a continuous space, the cells are confined to a grid. The size of the grid cells is equal to the security distance, which keeps the layout valid during the developmental process. The GRN is also trained with a genetic algorithm in order to produce the final layout. More details are given in [23]. The GRN has been trained with the fitness function from equation 2 on the same three training scenarios than DEvo-II.

\subsection{Results}

To evaluate Devo-II, we compare its results to layouts obtained using Devo-I, GA, PSO, and TDA on 10 different wind scenarios. Devo-I and Devo-II were exposed to 3 of the scenarios during training, but were run without exposure to the other 7. All scenarios are available in the supplementary materials and online. As both Devo-I and DEVO-II are deterministic once trained, the trained result was run only once on each scenario. The GA, PSO, and TDA were run 8 times on each scenario. Table 2 presents the best results for each method, and figure 4 presents the results of all runs.

First, we compare Devo-I and DEvo-II. The gain brought by the use of a continuous space is undeniable. The layouts

\footnotetext{
${ }^{2}$ Since TDA also uses Kusiak's wind model and after cross verifications, the layouts produced by TDA produce the same energy output as the layouts produced by our implementations
}

produced by DEvo-II are $7.9 \%$ better than the discrete ones, as the use of a continuous space allows Devo-II to locally optimize the positions of the same number of turbines.

Then, Devo-II and the GA are compared. With the same fitness function, DEvo-II does better on all scenarios: Devo-II uses more turbines but has a more efficient layout, getting a higher wake free ratio. Moreover, the number of evaluations is extremely low: 250,000 evaluations for the genetic algorithm in comparison to less than 100 evaluations for DEVO-II. The PSO again displays the advantages of a continuous model when compared to the GA, where it achieves better efficiencies with at least the same number of turbines. However, most likely due to constraint repairing mechanism [19], the PSO doesn't perform as well as DEVO-II or TDA. The number of evaluations necessary for the PSO, 2000 , is very costly compared to DEvo-II.

Compared to TDA with 2000 evalutions (TDA 2k), DEvoII produces solutions with a lower quality: the wake free ratio is $2.79 \%$ lower for Devo-II. However, Devo-II also optimizes the number of turbines, which is simply reused by TDA to optimize the layout.

To compare DEvo-II to TDA with comparable evaluation conditions, we run TDA with the number of evaluations used by DEvo-II. As shown in the column named "TDA devo" in table 2, DEvo-II produces equivalent solutions in quality to TDA using the number of turbines optimized and the number of evaluations used by DEvo-II.

We use a t-test to compare the results of each method with all others for each scenario. In these t-tests, only two from different methods have $p>0.05$ : in scenario 1, DEvoII and TDA $2 \mathrm{k}$ aren't significantly different, and in scenario 3, DEvo-II and TDA devo aren't significantly different. The t-test was performed over all 8 trials of each approach, where 2 only shows the best result from each trial.

These experiments demonstrate that Devo-II produces layouts that can compete with TDA in comparable conditions and outperforms GA and PSO. Moreover, DEVO-II can tackle a more complex problem than TDA: the optimization of both the number of turbines and their layout. This property can be useful in the design process of wind farms. In the conclusion, we will discuss the benefits and drawbacks of this approach in the wind farm design process.

\section{ADAPTATION TO THE ENVIRONMENT}

In our previous work using a discrete developmental model, DEvo-I, to produce the wind farm layouts [23], we briefly show that DEvo-I was able to adapt to changes in the layout size and to obstacles without retraining. Here, we offer a detailed study of Devo-II's ability to adapt to changes in the layout size and to obstacles.

\subsection{Adaptation to the layout size}

To show the capacity of Devo-II to adapt to the layout size, we produce layouts based on the previous wind scenarios but on a small layout $(6.2 \mathrm{~km}$ by $9.61 \mathrm{~km})$ and a large one $(9.92 \mathrm{~km}$ by $20.15 \mathrm{~km})$. DEVO-II is compared to Devo-I and TDA with 2000 evaluations. Table 3 shows the results of this study. Both Devo-I and Devo-II's results are deterministic, and TDA was run 8 times and the maximum fitness is showed in the table. As in the comparative study, TDA is run with the number of turbines given by DEVO-II.

The same conclusion as in $\S 7$ can be made when comparing these approaches, Devo-I, Devo-II, and TDA, on the 


\begin{tabular}{|c|ccc|ccc|ccc|ccc|ccc|ccc|}
\hline \multirow{2}{*}{ Scenario } & \multicolumn{3}{|c|}{ DEVO-II } & \multicolumn{3}{|c|}{ DEVO-I } & \multicolumn{3}{c|}{ GA } & \multicolumn{3}{c|}{ PSO } & \multicolumn{3}{c|}{ TDA 2k } & \multicolumn{2}{c|}{ TDA devo } \\
& $R_{w f}$ & $\# \mathrm{t}$ & $\# \mathrm{e}$ & $R_{w f}$ & $\# \mathrm{t}$ & $\# \mathrm{e}$ & $R_{w f}$ & $\# \mathrm{t}$ & $\# \mathrm{e}$ & $R_{w f}$ & $\# \mathrm{t}$ & $\# \mathrm{e}$ & $R_{w f}$ & $\# \mathrm{t}$ & $\# \mathrm{e}$ & $R_{w f}$ & $\# \mathrm{t}$ & $\# \mathrm{e}$ \\
\hline 0 & 0.880 & 403 & 100 & 0.825 & 399 & 100 & 0.873 & 399 & $250 k$ & 0.880 & 400 & 2000 & 0.901 & 403 & 2000 & 0.873 & 403 & 100 \\
1 & 0.934 & 408 & 100 & 0.862 & 461 & 100 & 0.927 & 400 & $250 k$ & 0.928 & 400 & 2000 & 0.936 & 408 & 2000 & 0.898 & 408 & 100 \\
2 & 0.870 & 400 & 100 & 0.825 & 406 & 100 & 0.858 & 400 & $250 k$ & 0.865 & 405 & 2000 & 0.890 & 400 & 2000 & 0.864 & 400 & 100 \\
3 & 0.857 & 400 & 59 & 0.794 & 405 & 100 & 0.838 & 400 & $250 k$ & 0.855 & 401 & 2000 & 0.892 & 400 & 2000 & 0.863 & 400 & 59 \\
4 & 0.853 & 399 & 58 & 0.791 & 466 & 100 & 0.837 & 399 & $250 k$ & 0.851 & 398 & 2000 & 0.893 & 399 & 2000 & 0.863 & 399 & 58 \\
\hline 5 & 0.881 & 405 & 100 & 0.833 & 403 & 100 & 0.871 & 399 & $250 k$ & 0.879 & 404 & 2000 & 0.908 & 405 & 2000 & 0.885 & 405 & 100 \\
6 & 0.892 & 400 & 100 & 0.781 & 627 & 100 & 0.880 & 400 & $250 k$ & 0.890 & 402 & 2000 & 0.912 & 400 & 2000 & 0.886 & 400 & 100 \\
7 & 0.875 & 402 & 61 & 0.780 & 479 & 100 & 0.879 & 400 & $250 k$ & 0.871 & 404 & 2000 & 0.909 & 402 & 2000 & 0.881 & 402 & 61 \\
8 & 0.890 & 409 & 62 & 0.822 & 523 & 100 & 0.879 & 400 & $250 k$ & 0.889 & 404 & 2000 & 0.911 & 409 & 2000 & 0.886 & 409 & 62 \\
9 & 0.891 & 401 & 57 & 0.848 & 424 & 100 & 0.874 & 400 & $250 k$ & 0.889 & 404 & 2000 & 0.913 & 401 & 2000 & 0.892 & 401 & 57 \\
\hline
\end{tabular}

Table 2: Maximum results of the comparative study of the wind farm layout optimization approaches. Italic values represents the parameters given to the algorithm, and thus not optimized. Columns are the wake free ratio $R_{w f}$, the number of turbines \# $\mathbf{t}$ and the number of layout evaluation \# $\mathrm{e}$ required to produce the layout.

small and large layouts: TDA achieves optimal results but of the same order of magnitude (on average, TDA is $2.76 \%$ better than Devo-II). This means Devo-II scales perfectly when the environment configuration changes; it performs the same relative to TDA as it does on the training layout. The number of evaluations is particularly crucial in this experiment, in particular on large layouts; whereas DEVO-II needs less than 4 minutes to produce the layouts, TDA $2 \mathrm{k}$ needs nearly one hour for each layout.

\subsection{Handling obstacles}

DEvo-II can naturally handle obstacles in the environment. This property is crucial since the wind farm design process has to account for possible obstacles such as lakes, roads, buildings, etc. In our approach, an obstacle is designed as a invalid position where the cells are forbidden to

\begin{tabular}{|l|l|ccc|ccc|ccc|}
\hline \multirow{2}{*}{$\mathrm{Sc}$} & Size & \multicolumn{2}{|c|}{ DEVO-II } & \multicolumn{3}{|c|}{ DEVO-I } & \multicolumn{3}{c|}{ TDA $2 \mathrm{k}$} \\
& & $R_{w f}$ & $\# \mathrm{t}$ & $\# \mathrm{e}$ & $R_{w f}$ & $\# \mathrm{t}$ & $\# \mathrm{e}$ & $R_{w f}$ & $\# \mathrm{t}$ & $\# \mathrm{e}$ \\
\hline 0 & small & 0.884 & 248 & 61 & 0.817 & 275 & 61 & 0.907 & 248 & 2000 \\
1 & small & 0.940 & 244 & 61 & 0.845 & 345 & 61 & 0.945 & 244 & 2000 \\
2 & small & 0.871 & 245 & 41 & 0.804 & 275 & 61 & 0.897 & 245 & 2000 \\
3 & small & 0.868 & 242 & 47 & 0.796 & 291 & 61 & 0.900 & 242 & 2000 \\
4 & small & 0.857 & 254 & 43 & 0.830 & 230 & 61 & 0.893 & 254 & 2000 \\
5 & small & 0.887 & 246 & 61 & 0.799 & 345 & 61 & 0.914 & 246 & 2000 \\
6 & small & 0.895 & 248 & 61 & 0.772 & 256 & 61 & 0.916 & 248 & 2000 \\
7 & small & 0.878 & 245 & 45 & 0.733 & 351 & 61 & 0.916 & 245 & 2000 \\
8 & small & 0.896 & 246 & 52 & 0.848 & 254 & 61 & 0.918 & 246 & 2000 \\
9 & small & 0.894 & 247 & 41 & 0.813 & 335 & 61 & 0.918 & 247 & 2000 \\
\hline 0 & large & 0.879 & 817 & 205 & 0.813 & 1013 & 205 & 0.890 & 817 & 2000 \\
1 & large & 0.922 & 833 & 205 & 0.865 & 1012 & 205 & 0.924 & 833 & 2000 \\
2 & large & 0.860 & 824 & 205 & 0.796 & 936 & 205 & 0.876 & 824 & 2000 \\
3 & large & 0.849 & 817 & 79 & 0.784 & 1012 & 205 & 0.881 & 817 & 2000 \\
4 & large & 0.847 & 841 & 87 & 0.808 & 786 & 205 & 0.876 & 841 & 2000 \\
5 & large & 0.878 & 817 & 87 & 0.817 & 1013 & 205 & 0.898 & 817 & 2000 \\
6 & large & 0.887 & 824 & 87 & 0.743 & 1013 & 205 & 0.900 & 824 & 2000 \\
7 & large & 0.869 & 819 & 81 & 0.793 & 1010 & 205 & 0.898 & 819 & 2000 \\
8 & large & 0.886 & 814 & 81 & 0.837 & 900 & 205 & 0.903 & 814 & 2000 \\
9 & large & 0.879 & 863 & 205 & 0.830 & 858 & 205 & 0.899 & 863 & 2000 \\
\hline
\end{tabular}

Table 3: Results of the modification of the farm size. Italic values represent the parameters given to the algorithm, and thus not optimized.

\begin{tabular}{|c|ccc|ccc|}
\hline \multirow{2}{*}{ Scenario } & \multicolumn{3}{|c|}{ No obstacle } & \multicolumn{3}{c|}{ Obstacles } \\
& $\begin{array}{c}\text { Size } \\
\left(\mathrm{km}^{2}\right)\end{array}$ & $\begin{array}{c}\text { Energy } \\
\text { per } \mathrm{km}^{2}\end{array}$ & $\begin{array}{c}\text { \# of } \\
\text { turb. }\end{array}$ & $\begin{array}{c}\text { Size } \\
\left(\mathrm{km}^{2}\right)\end{array}$ & $\begin{array}{c}\text { Energy } \\
\text { per km }\end{array}$ & $\begin{array}{c}\text { \# of } \\
\text { turb. }\end{array}$ \\
\hline 0 & 98 & 26,474 & 403 & 96.04 & 28,966 & 401 \\
1 & 98 & 54,640 & 408 & 96.04 & 54,813 & 402 \\
2 & 98 & 19,546 & 400 & 96.04 & 20,253 & 409 \\
3 & 98 & 24,506 & 400 & 96.04 & 25,241 & 404 \\
4 & 98 & 22,064 & 399 & 96.04 & 22,561 & 399 \\
\hline 5 & 98 & 32,333 & 405 & 96.04 & 32,558 & 399 \\
6 & 98 & 36,727 & 400 & 96.04 & 38,547 & 417 \\
7 & 98 & 32,730 & 402 & 96.04 & 32,542 & 391 \\
8 & 98 & 37,553 & 409 & 96.04 & 37,240 & 398 \\
9 & 98 & 38,460 & 401 & 96.04 & 39,573 & 406 \\
\hline
\end{tabular}

Table 4: Obstacle handling with Devo-II

go. Here again, no further training is necessary to take this new constraint into consideration: the evolved GRNs can directly produce layouts optimized with the obstacles.

To our knowledge, Devo-I and DEvo-II are the only approaches that currently tackle this problem. Therefore, to evaluate the quality of the generated layout, we propose a comparison of the energy output per kilometer square available in the environment. We use an initial $7 \mathrm{~km} \mathrm{x} 14 \mathrm{~km}$ layout size in this study. Table 4 presents the results of this comparison.

The energy per $\mathrm{km}^{2}$ is very comparable between the layout with and without obstacles. It only differs $2.4 \%$ on average on the 10 scenarios. This comparison is conclusive: DEvoII can adapt to obstacles in environment without modifying the properties of the layout it generates.

\section{CONCLUSION}

The paper presents a continuous developmental model, Devo-II, to optimize wind farm layouts. To evaluate this novel approach, we compare it to state-of-the-art techniques in layout optimization. We show that, in comparable conditions, DEVo-II generates layouts with comparable quality to those from state-of-the-art techniques. However, DevoII optimizes both the number of turbines and their layout, and can adapt to handle changing layout size and obstacles without retraining. These properties are crucial during the design process of a wind farm. 
The main benefit of this approach is its immediate reactivity which is not found in other approaches. The developmental model only needs a few seconds to adapt to new constraints. Therefore, Devo-II could be an helpful tool for wind farm designers, bearing in mind that the layout process requires a great deal of auxillary input guidance from engineers. DEvo-II could be used as a real-time optimization tool during this design phase. Designers could interact with the cells, constraints, etc. while the cells are optimizing the layout.

Of course, because Devo-II doesn't currently provide the optimal layout, this tool could be used along with a standard layout optimizer. TDA could be used, for example, to precisely position the final turbines generated by DEVO-II. Our hypothesis is that TDA could optimize this layout faster and potentially better because of DEvo-II, which provides the number of turbines and an efficient starting layout.

\section{Acknowledgement}

This work was granted access to the HPC resources of CALMIP under the allocation 2013-1319

\section{REFERENCES}

[1] S. Chowdhury, J. Zhang, A. Messac, and L. Castillo. Unrestricted wind farm layout optimization (uwflo): Investigating key factors influencing the maximum power generation. Renewable Energy, 38(1):16-30, 2012.

[2] S. Cussat-Blanc, J. Pascalie, S. Mazac, H. Luga, and Y. Duthen. A synthesis of the Cell2Organ developmental model. Morphogenetic Engineering, 2012.

[3] S. Cussat-Blanc, S. Sanchez, and Y. Duthen. Controlling cooperative and conflicting continuous actions with a gene regulatory network. In Conference on Computational Intelligence in Games (CIG'12). IEEE, 2012.

[4] E. H. Davidson. The regulatory genome: gene regulatory networks in development and evolution. Academic Press, 2006.

[5] R. Doursat. Organically grown architectures: Creating decentralized, autonomous systems by embryomorphic engineering. Organic Computing, pages 167-200, 2008.

[6] A. Emami and P. Noghreh. New approach on optimization in placement of wind turbines within wind farm by genetic algorithms. Renewable Energy, 35(7):1559-1564, 2010.

[7] S. Grady, M. Hussaini, and M. M. Abdullah. Placement of wind turbines using genetic algorithms. Renewable Energy, 30(2):259-270, 2005.

[8] P. E. Hotz. Genome-physics interaction as a new concept to reduce the number of genetic parameters in artificial evolution. In Evolutionary Computation, 2003. CEC'03. The 2003 Congress on, volume 1 , pages 191-198. IEEE, 2003.

[9] H.-S. Huang. Distributed genetic algorithm for optimization of wind farm annual profits. In Intelligent Systems Applications to Power Systems, 200\%. ISAP 200\%. International Conference on, pages 1-6. IEEE, 2007.

[10] M. Joachimczak and B. Wróbel. Evo-devo in silico: a model of a gene network regulating multicellular development in $3 \mathrm{~d}$ space with artificial physics. In Proceedings of the 11th International Conference on Artificial Life, pages 297-304. MIT Press, 2008.

[11] M. Joachimczak and B. Wróbel. Evolving Gene Regulatory Networks for Real Time Control of Foraging Behaviours. In Proceedings of the 12th International Conference on Artificial Life, 2010.

[12] S. A. Khan and S. Rehman. Iterative non-deterministic algorithms in on-shore wind farm design: A brief survey. Renewable and Sustainable Energy Reviews, 19:370-384, 2013.

[13] V. Komkov. Optimal control theory for the damping of vibrations of simple elastic systems. Springer-Verlag Berlin, 1972.

[14] A. Kusiak and Z. Song. Design of wind farm layout for maximum wind energy capture. Renewable Energy, 35(3):685-694, 2010.

[15] G. Mosetti, C. Poloni, and B. Diviacco. Optimization of wind turbine positioning in large windfarms by means of a genetic algorithm. Journal of Wind Engineering and Industrial Aerodynamics, 51(1):105-116, 1994.

[16] M. Nicolau, M. Schoenauer, and W. Banzhaf. Evolving genes to balance a pole. Genetic Programming, pages 196-207, 2010.

[17] B. Porter. A developmental system for organic form synthesis. In Artificial Life: Borrowing from Biology, pages 136-148. Springer, 2009.

[18] S. Şişbot, Ö. Turgut, M. Tunç, and Ü. Çamdalı. Optimal positioning of wind turbines on gökçeada using multi-objective genetic algorithm. Wind Energy, 13(4):297-306, 2010.

[19] K. Veeramachaneni, M. Wagner, U.-M. O'Reilly, and F. Neumann. Optimizing energy output and layout costs for large wind farms using particle swarm optimization. In Evolutionary Computation (CEC), 2012 IEEE Congress on, pages 1-7. IEEE, 2012.

[20] M. Wagner, J. Day, and F. Neumann. A fast and effective local search algorithm for optimizing the placement of wind turbines. Renewable Energy, 51(0):64 - 70, 2013.

[21] C. Wan, J. Wang, G. Yang, X. Li, and X. Zhang. Optimal micro-siting of wind turbines by genetic algorithms based on improved wind and turbine models. In Decision and Control, 2009 held jointly with the 2009 28th Chinese Control Conference. $C D C / C C C$ 2009. Proceedings of the 48th IEEE Conference on, pages 5092-5096. IEEE, 2009.

[22] C. Wan, J. Wang, G. Yang, and X. Zhang. Optimal micro-siting of wind farms by particle swarm optimization. In Advances in swarm intelligence, pages 198-205. Springer, 2010.

[23] D. Wilson, E. Awa, S. Cussat-Blanc, K. Veeramachaneni, and U.-M. O'Reilly. On learning to generate wind farm layouts. In Proceeding of the fifteenth annual conference on Genetic and evolutionary computation conference. ACM, 2013.

[24] C. Xu, Y. Yan, D. Y. Liu, Y. Zheng, and C. Q. Li. Optimization of wind farm micro sitting based on genetic algorithm. Advanced Materials Research, 347:3545-3550, 2012. 